\title{
Community compulsion in Scotland: a descriptive analysis
}

\author{
Rachel Brown, ${ }^{1}$ Mark Taylor, ${ }^{2}$ Kirsty Mackay, ${ }^{1}$ Callum Macleod, ${ }^{3}$ Donald Lyons ${ }^{3}$
}

The Psychiatrist (2012), 36, 205-207, doi: 10.1192/pb.bp.110.033761

${ }^{1}$ Ballenden House, Edinburgh,

${ }^{2}$ Henderson Unit, Royal Edinburgh Hospital; ${ }^{3}$ Mental Welfare Commission for Scotland, Edinburgh

Correspondence to Mark Taylor (mark.taylor2@nhs.net)

First received 3 Dec 2011, final revision 7 Sep 2011, accepted 10 Nov 2011

\begin{abstract}
Aims and method Community compulsion via community compulsory treatment orders is used routinely in Scotland. We aimed to describe the common characteristics of individuals subject to community compulsion. We collected standardised information from a national database about individuals subject to community compulsion and compared them with people subject to hospital detention.
\end{abstract}

Results Analysis of 499 cases revealed that the majority of individuals subject to community compulsion had a psychotic illness, had a history of non-adherence to services and treatment, and were more likely than not to be in receipt of a long-acting injection of antipsychotic medication. Patients subject to community compulsion were clinically similar to patients subject to hospital-based treatment orders and usually were considered to pose a risk to other people.

Clinical implications Community compulsion has been widely adopted despite a relative lack of supporting scientific evidence. Our findings are similar to those of other related studies and highlight that individuals with a psychotic illness who are ambivalent about treatment and who pose a risk to self or others are likely to be considered for community compulsion.

Declaration of interest None.
Compulsory treatment in the community came into force in Scotland in October 2005 via community compulsory treatment orders (CCTOs) under the Mental Health (Care $\&$ Treatment) (Scotland) Act 2003 (the Act). In England and Wales, similar community compulsion occurs via community treatment orders, which were introduced in 2008 under the 2007 amendments of the Mental Health Act 1983. Community compulsory treatment orders can authorise a number of powers, the extent of which are decided on an individual basis by the Mental Health Tribunal for Scotland; they include not only compulsory medical treatment but also a requirement to attend appointments or to reside at a certain address. Criteria for community compulsory treatment are identical to those for hospital compulsory treatment namely that the patient has a mental disorder; that there is treatment available to prevent the disorder worsening or to alleviate symptoms or effects of the disorder; that without treatment there would be significant risk to the health, safety or welfare of the patient or safety of others; that the patient has significantly impaired decision-making ability in relation to treatment decisions (regarding their mental health); and that the making of a CCTO is necessary. Rates of CCTO granted by the Tribunal increased steadily following introduction of the Act until 2007 and now represent approximately a third of all long-term treatment orders in Scotland. ${ }^{1}$

Despite this rate of uptake of CCTOs, community compulsion remains a controversial subject. A Cochrane review that considered two international randomised controlled trials (but excluded other qualitative research) concluded that CCTOs may not be an effective alternative to standard care. $^{2}$ Opponents of compulsory community treatment cite control and threat as reasons to oppose such measures, arguing that compulsory community treatment may adversely affect the therapeutic alliance and drive people with severe mental illness away from services. ${ }^{3}$ Randomised controlled trials of community compulsion have been criticised for their lack of generalisability due to substantial differences in service structure and function. ${ }^{4}$

Little is known about the effectiveness of compulsory measures in the community in the UK. At the time of writing, results are awaited from the Oxford Community Treatment Order Evaluation Trial.

In this study, we aim to describe the population subject to CCTOs 2 years after introduction of the Act.

\section{Method}

The Mental Welfare Commission for Scotland is required to maintain a case register database of all episodes of detention under the Act. All individuals who were subject to a CCTO on 8 August 2007 were identified from this database. This date was chosen to be representative of the steady-state national rate use of CCTOs, as the rate of CCTO use had risen steadily from October 2005 until that time and then reached a relative plateau. The date was also 
chosen because a separate related study needed sufficient time for further research.

Individual case data were anonymised and collected using a standardised pro forma questionnaire that was devised following literature review and consultation among the authors. We analysed each case record held at the Mental Welfare Commission for Scotland by extracting data from Act forms and matching each record against the standardised pro forma. Data were recorded in a spreadsheet. The pro forma questionnaire included the following variables: diagnosis, social situation, treatment authorised, medication, detention criteria, and reasons stated for CCTO. Diagnosis was recorded according to the diagnosis specified in the case data; where no ICD-10 diagnosis was specified, we categorised the diagnosis based on the clinical information detailed within the individual case data.

To compare the population subject to CCTOs with the population subject to hospital compulsory treatment, all people subject to hospital compulsory treatment on 8 August 2007 were sampled and data regarding their risk status and diagnosis were gathered.

\section{Results}

A total of 499 individuals were subject to CCTO in Scotland on 8 August 2007. Of these people, $63 \%$ were male; the majority were living alone (63\%) with a diagnosis of schizophrenia (70\%), schizoaffective disorder (10\%) or bipolar disorder (10\%); $97.4 \%$ had mental illness; $8 \%$ had intellectual disability (defined in the Act as 'learning disability'); and $6 \%$ had a listed ICD-10 comorbid diagnosis of polysubstance misuse, with $5 \%$ documented as misusing cannabis and $1 \%$ misusing or being dependent on alcohol. All 499 individuals (100\%) were deemed to lack insight into their treatment needs (which is unsurprising, given the criteria for significantly impaired decision-making ability in the Act), and the vast majority had a history of nonadherence with medication or services (96\% and 98\%, respectively), as detailed in the Act forms. Of the total sample, $99 \%$ were considered to pose a risk to themselves and $65 \%$ were considered to pose a risk to others.

Fifty-eight per cent of the total sample were receiving regular treatment with antipsychotic long-acting injections, and just under half of the total sample (48\%) were either refusing to consent to treatment or deemed unable to consent to treatment. Of the 499 individuals, 34\% were prescribed regular multiple antipsychotics (Tables 1-3).

In comparison, 1373 individuals were subject to hospital community treatment in Scotland on 8 August 2007. Of these people, $94.5 \%$ had a diagnosis of mental illness and $13.4 \%$ intellectual disability; $7.4 \%$ (v. $2.6 \%$ of the CCTO sample) had a diagnosis of personality disorder; $73 \%$ ( $v .65 \%$ of the CCTO sample) were considered to pose a risk to others; and $99.8 \%$ were considered to pose a risk to self (Tables 4 and 5).

\section{Discussion}

This study provides an insight into the patient population subject to CCTOs after the rate of uptake of such community measures had reached a steady state. This national sample should be generalisable to other jurisdictions
Table 1 Social circumstances of community compulsory treatment order sample

\begin{tabular}{lrr} 
Social circumstances & $n$ & $\%$ \\
\hline Living alone & 248 & 49.7 \\
\hline Living alone with support service input & 65 & 13.0 \\
\hline Supported accommodation & 65 & 13.0 \\
\hline Living with family & 107 & 21.4 \\
\hline Other, including residential care & 14 & 2.8 \\
\hline
\end{tabular}

Table 2 Antipsychotic treatments prescribed in community compulsory treatment order sample

\begin{tabular}{lcc} 
Antipsychotic treatment & $n$ & $\%$ \\
\hline Clozapine & 72 & 14.4 \\
\hline Depot/long-acting injection & 287 & 57.5 \\
\hline One antipsychotic & 279 & 55.9 \\
\hline Two antipsychotics & 161 & 32.3 \\
\hline Three antipsychotics & 6 & 1.2 \\
\hline Not known (data missing) & 43 & 8.6 \\
\hline
\end{tabular}

Table 3 Other medications prescribed in community compulsory treatment order sample

\begin{tabular}{lcr} 
Medication & $n$ & $\%$ \\
\hline Antidepressant & 85 & 17.0 \\
\hline Mood stabiliser & 99 & 19.8 \\
\hline Anxiolytic & 210 & 42.1 \\
\hline Hypnotic & 33 & 6.6 \\
\hline
\end{tabular}

Table 4 Risk status in hospital-based and community compulsory treatment order samples

\begin{tabular}{lcc} 
Risk & $\begin{array}{c}\text { Hospital } \\
n(\%)\end{array}$ & $\begin{array}{c}\text { Community } \\
n(\%)\end{array}$ \\
\hline Danger to others & $1(0.1)$ & $1(0.2)$ \\
\hline Danger to self & $372(27.1)$ & $172(34.5)$ \\
\hline Danger to self and others & $998(72.7)$ & $325(65.1)$ \\
\hline None recorded & $2(0.1)$ & $1(0.2)$ \\
\hline Sample total & 1373 & 499 \\
\hline
\end{tabular}

with similar compulsory measures, such as requirements to accept treatment, to attend appointments and to notify professionals before any change of address. This study illustrates that the profile of an individual subject to community compulsion is often a man with a psychotic illness, living alone with a history of non-adherence to medication and services, and in receipt of long-acting injectable antipsychotic medication. This is in keeping with findings by the Institute of Psychiatry, which, following a review of 14 studies across multiple jurisdictions, concluded in its 2007 report that the characteristics of patients subject to CCTO were 'remarkably consistent'. ${ }^{5}$ Interestingly, the 


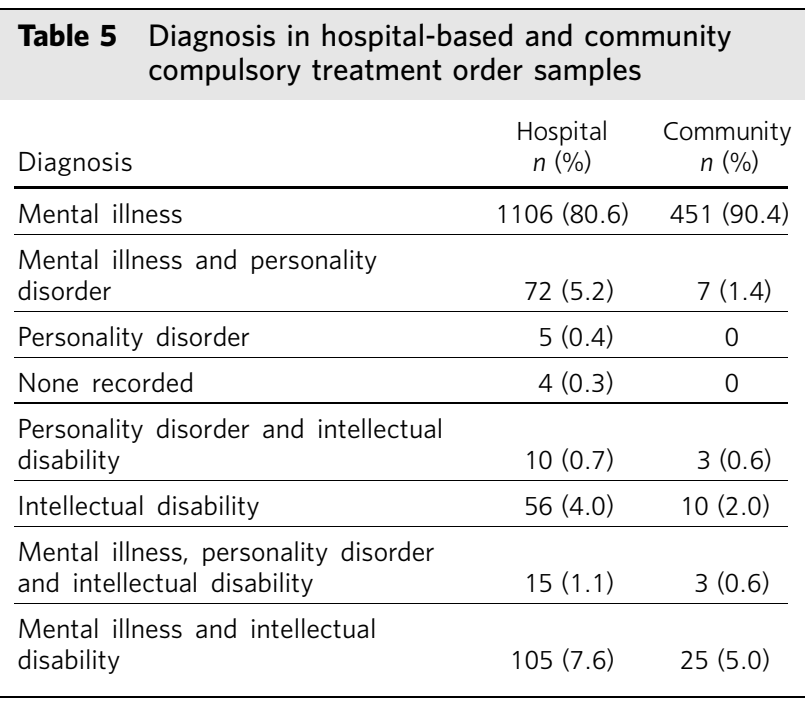

profile of individuals subject to hospital-based compulsory treatment in August 2007 was strikingly similar to that of individuals subject to CCTOs.

Almost half of the sample were assessed as lacking capacity to consent or were actively refusing medical treatment. Every patient was deemed to lack insight into their mental disorder or need for treatment. Of the CСТO sample, $65 \%$ were deemed to pose a risk to others (in addition to self), which may indicate that clinicians are more likely to use community treatment orders in situations where risk to others is a concern but hospitalisation is not required, and adherence to medical treatment is considered necessary to minimise that risk. ${ }^{6}$ This is suggested by a recent study of community treatment orders in Birmingham and Solihull, in which reported rates of previous violence in their sample reached $92.3 \%{ }^{7}$ Evans and colleagues comment that few studies have reported on this area, ${ }^{7}$ but a study by Swanson and colleagues reported $51 \%$ of their sample had a history of violence shortly before use of a community treatment order. ${ }^{8}$ Studies from New Zealand, however, have reported much lower rates of any history of aggression in their sample (38.2\%). ${ }^{9}$ A review of 11 reports of non-randomised comparative studies of community treatment order outcomes concluded that community treatment orders had no real effect on social functioning, violence, disturbed behaviour or arrest. $^{5}$

Community treatment orders remain controversial, despite their widespread uptake, as indicated by a Cochrane review that concluded that 85 people would need to be subject to community compulsory treatment to avoid 1 psychiatric admission, and that 238 community treatment orders would be required to avoid 1 arrest. ${ }^{3}$ Additionally, a systematic review by Churchill and colleagues concluded that community orders lacked consistent evidence of benefit. ${ }^{5}$ Qualitative data were excluded from the Cochrane review, however, and some authors have concluded that community compulsory treatment does have benefits; for example, O'Reilly and colleagues, who found that patients thought community compulsion provided structure in their lives, families found community compulsion to be necessary when patient insight was limited, and clinicians 'were more consistently positive' than either patients or families. ${ }^{10}$ This may reflect negative past experience of hospital on the part of the patient or reflect views at a time when patients have gained insight into their treatment needs.

Herein lies the paradox in the use of community treatment orders - international research evidence to date is contradictory but suggests that community compulsory treatment is no more effective than standard care, and yet clinicians continue to favour use of community treatment orders in arguably the more challenging clinical populations. Clearly there remains a need for further research into the effect of community treatment orders on a range of different outcomes to evidence and guide their use in everyday practice. In particular, it would be relevant to examine the impact of community treatment orders on hospital bed days and admission rates, suicide rates and patients' level of functioning, and for further qualitative research into attitudes towards community treatment orders. At the time of writing, a separate study is being pursued to research these issues.

\section{About the authors}

Rachel Brown is a consultant psychiatrist at Ballenden House, Edinburgh. Mark Taylor is a consultant psychiatrist at the Henderson Unit, Royal Edinbugh Hospital. Kirsty Mackay is a consultant psychiatrist at Ballenden House, Edinburgh. Callum Macleod is a systems analyst and Donald Lyons is chief executive at the Mental Welfare Commission for Scotland.

\section{References}

1 Mental Welfare Commission for Scotland. Total Number of Orders in Existence. Mental Welfare Commission for Scotland, 2008.

2 Kisely S, Campbell LA, Preston N. Compulsory community and involuntary outpatient treatment for people with severe mental disorders. Cochrane Database Syst Rev 2005; 3: CD004408.

3 Pinfold V, Bindman J. Is compulsory community treatment ever justified? Psychiatrist 2001; 25: 268-70.

4 Lambert TJ, Singh BS, Patel MX. Community treatment orders and antipsychotic long-acting injections. Br J Psychiatry 2009; 95 (suppl 52): s57-62.

5 Churchill R, Owen G, Singh S, Hotopf M. International Experiences of Using Community Treatment Orders. UK Department of Health and Institute of Psychiatry, 2007.

6 Taylor M. Community treatment orders and their use in the UK. Adv Psychiatr Treat 2010; 16: 260-2.

7 Evans R, Makala J, Humphreys M, Mohan CRN. Supervised community treatment in Birmingham and Solihull: first 6 months. Psychiatrist 2010; 34: $330-3$.

8 Swanson JW, Swartz MS, Wagner HR, Burns BJ, Borum R, Hiday VA Involuntary out-patient commitment and reduction of violent behaviour in persons with severe mental illness. Br J Psychiatry 2000; 176: 324-31.

9 Dawson J, Romans S. Uses of community treatment orders in New Zealand: early findings. Aust N Z J Psychiatry 2001; 35: 190-5.

10 O'Reilly R, Keegan DL, Corring D, Shrikhande S, Natarajan D. A qualitative analysis of the use of community treatment orders in Saskatchewan. Int J Law Psychiatry 2006; 29: 516-24. 